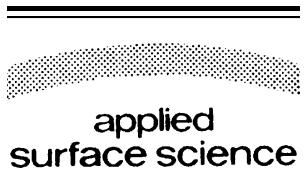

Applied Surface Science 246 (2005) 377-380

www.elsevier.com/locate/apsusc

\title{
Mixed Langmuir monolayers of an amphiphilic chromo-ionophore and the phospholipid DMPC
}

\author{
T.I. Sergeeva ${ }^{a}$, S.P. Gromov ${ }^{b}$, A.I. Vedernikov ${ }^{b}$, M.S. Kapichnikova ${ }^{b}$, \\ M.V. Alfimov ${ }^{\mathrm{b}}$, D. Möbius ${ }^{\mathrm{c}, *}$, S.Yu. Zaitsev ${ }^{\mathrm{a}, \mathrm{d}}$ \\ ${ }^{a}$ Institute of Bioorganic Chemistry, Russian Academy of Sciences, Miklukho-Maklaya Str. 16/10, Moscow 117871, Russia \\ ${ }^{\mathrm{b}}$ Center Photochemistry, Russian Academy of Sciences, Novatorov Str. 7a, Moscow 117334, Russia \\ ${ }^{\mathrm{c}}$ Max-Planck-Institut für biophysikalische Chemie, D-37070 Göttingen (F.R.G.), Russia \\ ${ }^{\mathrm{d}}$ Department of Organic and Biological Chemistry, Moscow State Academy of Veterinary Medicine and Biotechnology, \\ Acad. Skryabina Str. 23, Moscow 109472, Russia
}

Available online 4 January 2005

\begin{abstract}
The amphiphilic chromo-ionophore 2-[(1E,3E)-4-(2,3,5,6,8,9,11,12,14,15-decahydro-1,7,10,16,4,13-benzotetraoxadithiacyclooctadecin-18-yl)-1,3-butadienyl]-3-octadecyl-1,3-benzothiazol-3-ium perchlorate (AB211) forms mixed monolayers at the air-water interface with the phospholipid L- $\alpha$-dimyristoylphosphatidylcholine (DMPC) as deduced from surface pressurearea and surface potential-area isotherms. In contrast to the behaviour of pure monolayers of AB211 on water, no association of the chromophores is observed by reflection spectroscopy. Upon compression of the mixed monolayer AB211:DMPC $=1: 5$, a reorientation of the chromophores from flat to tilted is observed, presumably assisted by the stretching of the choline group of the phospholipid molecules acting a matrix.
\end{abstract}

(C) 2004 Elsevier B.V. All rights reserved.

Keywords: Langmuir monolayer; Chromo-ionophore; Amphiphilic

\section{Introduction}

Amphiphilic chromo-ionophores are of particular interest since they combine three functions: (a)

Abbreviations: AB211, 2-[(1E,3E)-4-(2,3,5,6,8,9,11,12,14,15decahydro-1,7,10,16,4,13-benzotetraoxadithiacyclooctadecin-18yl)-1,3-butadienyl]-3-octadecyl-1,3-benzothiazol-3-ium perchlorate; DMPC, $\mathrm{L}-\alpha$-dimyristoylphosphatidylcholine

* Corresponding author. Tel.: +49 5512011364 ; fax: +49 551201108 .

E-mail address: dmoebiu@gwdg.de (D. Möbius). formation of monolayers; (b) selective complexation of cations; (c) photoreactivity [1-10]. Langmuir monolayers of the chromo-ionophore 2-[(1E,3E)4-(2,3,5,6,8,9,11,12,14,15-decahydro-1,7,10,16,4,13benzotetraoxadithiacyclooctadecin-18-yl)-1,3-butadienyl]-3-octadecyl-1,3-benzothiazol-3-ium perchlorate (AB211) (structure, see Fig. 1) show unusual association phenomena, as well as interactions with various cations and anions in the subphase [11]. In order to facilitate the assignment of the numerous bands observed in the reflection spectrum of pure 
AB211 monolayers on water, it is necessary to measure the spectrum of the monomer at the air-water interface. Further, such measurements may provide information on the orientation of the transition moments at the air-water interface. A simple strategy to approach this goal is the formation of twocomponent monolayers with AB211 embedded in an appropriate matrix. Monolayers with chromoionophore monomers are of particular practical interest since irreversible photo-dimer formation is avoided, and reversible cis-trans isomerization the only photo-transformation besides irreversible processes leading to photo-degradation. Reversible photo-isomerization may enable one to switch complexation of the ionophore. Such controlled reversible complex formation is potentially useful for waste water treatment and metal ion enrichment. Here, we report on the study of two-component monolayers using the phospholipid $\mathrm{L}$ - $\alpha$-dimyristoylphosphatidylcholine (DMPC) as matrix.

\section{Experimental}

\subsection{Materials}

The benzo-dithia-18-crown-6 butadienyl dye AB211 has been prepared by condensation of 2methyl-3-octadecyl-benzthiazolium perchlorate with dithia-18-crown-6 ether cinamylaldehyde [11]. The phospholipid L- $\alpha$-dimyristoylphosphatidylcholine $(99 \%+)$ was purchased from Sigma and used without further purification. The water has been cleaned with a Milli-Q filtration unit of Millipore Corp. (specific resistance: $18 \mathrm{M} \Omega \mathrm{cm}$, surface tension: $72.7 \mathrm{mN} / \mathrm{m}$ at $\left.20{ }^{\circ} \mathrm{C}\right)$.

\subsection{Methods}

The surface pressure $(\pi)$-molecular area $(A)$ and surface potential $(\Delta V)$-molecular area isotherms of dye monolayers were recorded using a rectangular trough (dimensions: $11 \mathrm{~cm} \times 36 \mathrm{~cm} \times \mathrm{cm}$ ) made from poly(tetrafluoroethylene) equipped with a $15 \mathrm{~mm}$ wide filter paper Wilhelmy balance and vibrating plate condenser (with Pt plate, operating at $400 \mathrm{~Hz}, 1.5 \mathrm{~cm}$ diameter). A volume of about $35 \mu \mathrm{L}$ of the $1 \mathrm{mM}$ solutions of AB211, DMPC, and of mixtures AB211:DMPC, respectively, in chloroform was spread onto water $\left(21^{\circ} \mathrm{C}\right)$, and after 10 min relaxation the monolayers were compressed by moving the barrier with constant speed reducing the surface area by about $16.1 \mathrm{~cm}^{2} / \mathrm{min}$. Reflection spectra of the mixed monolayers at constant surface pressure were measured under normal incidence of light with a modified spectrometer of the type described earlier [12] and plotted as the difference $\Delta R_{\mathrm{n}}$ of the reflectivities of the monolayer-covered surface and the bare water surface, normalized with respect to the surface density of $\mathrm{AB} 211$.

\section{Results and discussion}

Surface pressure-area, $\pi-A$ (top), and surface potential-area, $\Delta V-A$ (bottom), isotherms of Langmuir monolayers of the pure AB211 (curve 1), DMPC (curve 2), and of the mixture AB211:DMPC $=1: 5$ (curve 3) are shown in Fig. 1. It is obvious that the isotherms of the mixture (curve 3) differ from the average that may be calculated using the isotherms of the pure components (i.e. no additivity of the molecular area or the surface potential). This is an evidence of mixing of the components in the Langmuir monolayer at the air-water interface. It is remarkable that the surface potential of the twocomponent monolayer AB211:DMPC $=1: 5$ is much more positive than that of the pure components for areas $A<1 \mathrm{~nm}^{2}$, when the surface pressure is starting to increase upon compression. This points to a particular organization of the monolayer, possibly with a larger separation of the counter ion of AB211 from the positively charged benzothiazolium part of the chromophore.

The average area per molecule taken from the $\pi-A$ isotherms in Fig. 1 and others (not shown) at the ratios $1: 1,1: 3$, and $1: 8$ are plotted versus the molar fraction of AB211 in Fig. 2 for the surface pressures $10 \mathrm{mN} / \mathrm{m}$ (squares) and $20 \mathrm{mN} / \mathrm{m}$ (circles). The straight lines represent an ideal behaviour, and the deviations from such behaviour are clearly seen. Therefore, the phospholipid DMPC seems to be a matrix for AB211 that may inhibit molecular association of the chromo-ionophore molecules in Langmuir monolayers. 


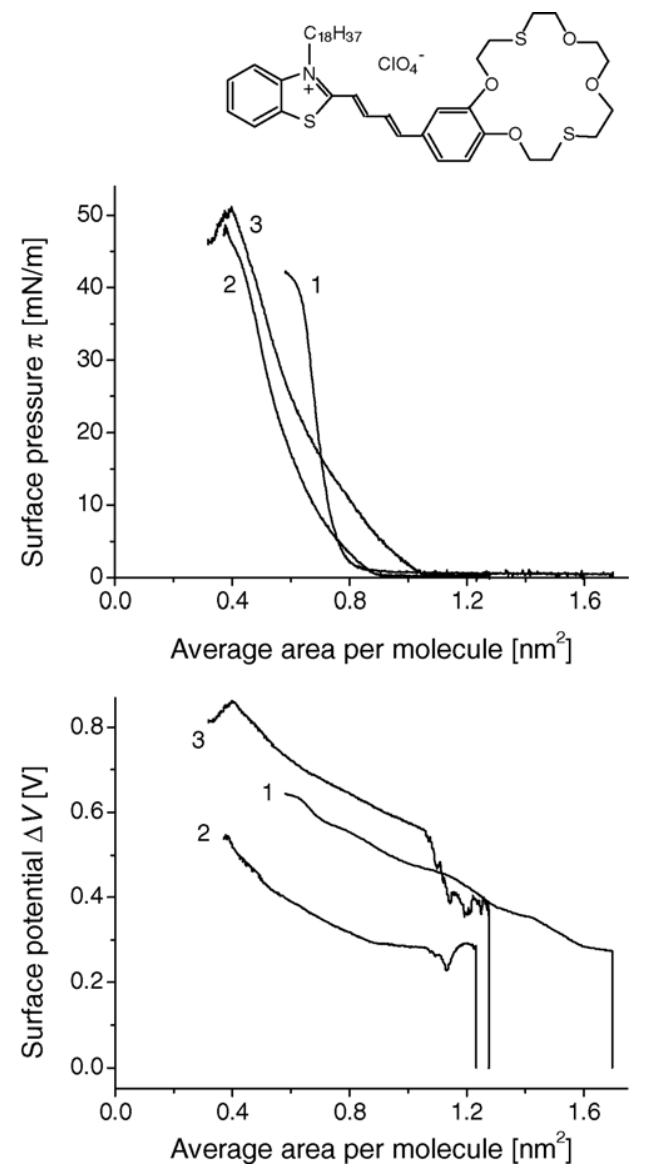

Fig. 1. Surface pressure-area ( $\pi-A$, top) surface potential-area $(\Delta V-$ $A$, bottom) isotherms at the air-water interface of (1) AB211; (2) DMPC; (3) AB211:DMPC $=1: 5 ; 20{ }^{\circ} \mathrm{C}$.

Molecular association in Langmuir monolayers is easily detected by measuring reflection spectra. Fig. 3 shows the reflection specta $\Delta R_{\mathrm{n}}(\lambda)$ of the monolayer $\mathrm{AB} 211: \mathrm{DMPC}=1: 5$ at the air-water interface at the surface pressures $1,5,10,15$, and $20 \mathrm{mN} / \mathrm{m}$ (from top to bottom). For comparison, the absorption spectrum of AB211 in solution (solvent acetonitrile, concentration $5.74 \times 10^{-5} \mathrm{M} ; 2 \mathrm{~mm}$ cuvette) is also shown. The reflection spectra have been normalized with respect to the surface density of AB211. The reflection spectrum measured at $1 \mathrm{mN} / \mathrm{m}$ closely resembles that of the solution. Therefore, the chromo-ionophore molecules do not associate in the mixed monolayer with DMPC as a matrix, in contrast to the behavior in pure AB211 monolayers. With increasing surface

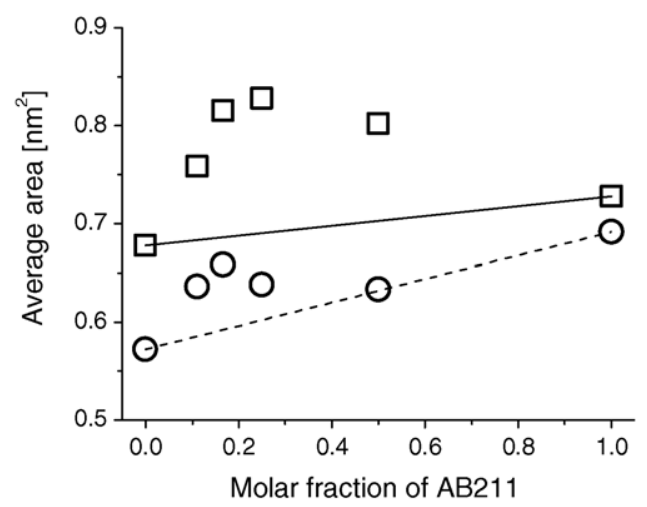

Fig. 2. Average areas at $10 \mathrm{mN} / \mathrm{m}$ (squares) and $20 \mathrm{mN} / \mathrm{m}$ (circles) of monolayer at the air-water interface of AB211, DMPC, and $\mathrm{AB} 211: \mathrm{DMPC}=1: r, r=1,3,5,8$ plotted vs. molar fraction of AB211; clear deviations from ideal behavior (indicated by the straight lines); $20{ }^{\circ} \mathrm{C}$.

pressure, the maximum value of $\Delta R_{\mathrm{n}}$ is decreasing, and the wavelength of the maximum is shifted from $465 \mathrm{~nm}(1 \mathrm{mN} / \mathrm{m})$ to $452 \mathrm{~nm}(20 \mathrm{mN} / \mathrm{m})$. The shift to shorter waves is attributed to a small fraction of AB211 forming dimers [13]. The reduction of the maximum signal must be attributed to a chromophore tilt. Indeed, the value of $\Delta R_{\mathrm{n}}$, measured at $1 \mathrm{mN} / \mathrm{m}$, coincides with that calculated from the extinction coefficient in solution and the area per AB211, assuming statistical orientation of the transition moment in the layer plane [14]. Disregarding partial

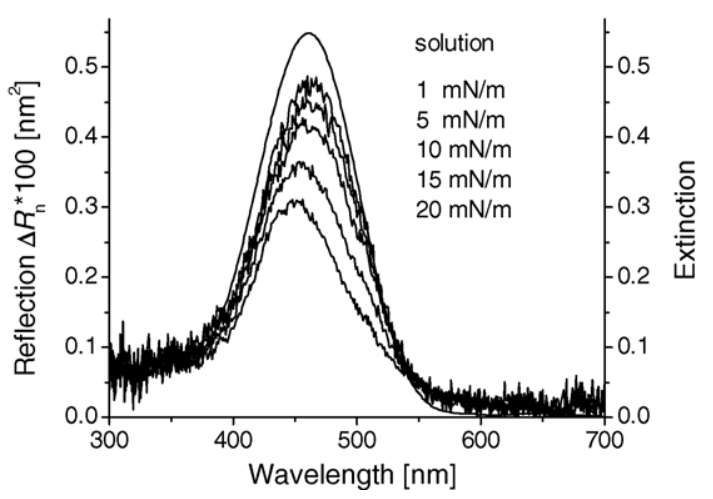

Fig. 3. Absorption spectrum of AB211 in solution (solvent acetonitrile, $5.74 \times 10^{-5} \mathrm{M} ; 2 \mathrm{~mm}$ ) and reflection spectra of the monolayer $\mathrm{AB} 211$ :DMPC $=1: 5$ at the air-water interface at different surface pressures indicated; the reflection spectra were normalized to same surface density of AB211 according to $\Delta R_{\mathrm{n}}=\Delta R_{\text {measured }} A_{\mathrm{AB} 211}$. 


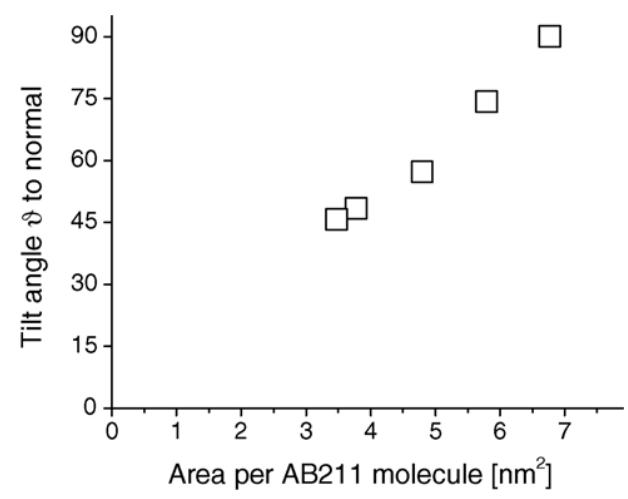

Fig. 4. Average tilt angle of transition moments with respect to the surface normal plotted vs. area per AB211 in the mixed monolayer $\mathrm{AB} 211 \mathrm{DMPC}=1: 5$ as determined from the maximum value of normalized reflection taken from Fig. 3 .

formation of dimers, an average angle, $\vartheta$, of the transition moments to the surface normal has been calculated using the maximum values of $\Delta R_{\mathrm{n}}$ and is plotted versus the area per AB211 in the mixed monolayer in Fig. 4. The angle $\vartheta$ decreases from $90^{\circ}$ (flat lying chromophores) at $1 \mathrm{mN} / \mathrm{m}$ to about $45^{\circ}$ at $20 \mathrm{mN} / \mathrm{m}$.

\section{Conclusion}

The chromo-ionophore AB211 forms mixed monolayers at the air-water interface with the phospholipid DMPC. Monomers of AB211 are observed at low surface pressures. With increasing surface pressure, the chromophores assume a tilted orientation and dimers are formed. These results may facilitate the understanding of the complex behavior of AB211 monolayers observed on water and various salt solutions.

\section{References}

[1] I.K. Lednev, M.C. Petty, Langmuir 10 (1994) 4185-4189.

[2] I.K. Lednev, M.C. Petty, Langmuir 10 (1994) 4190-4194.

[3] I.K. Lednev, M.C. Petty, J. Phys. Chem. 99 (1995) 4176-4180.

[4] I.K. Lednev, M.C. Petty, Adv. Mater. 8 (1996) 615-630.

[5] S.Y. Zaitsev, V.P. Vereschetin, S.P. Gromov, O.A. Fedorova, M.V. Alfimov, H. Huesmann, D. Möbius, Supramol. Sci. 4 (1997) 519-524.

[6] S.Y. Zaitsev, S.P. Gromov, O.A. Fedorova, E.A. Baryshnikova, V.P. Vereschetin, W. Zeiß, H. Huesmann, M.V. Alfimov, D. Möbius, Colloids Surf. A 131 (1998) 325-332.

[7] S.Y. Zaitsev, V.P. Vereschetin, E.A. Baryshnikova, S.P. Gromov, O.A. Fedorova, M.V. Alfimov, H. Huesmann, D. Möbius, Thin Solid Films 329 (1998) 821-823.

[8] S.Y. Zaitsev, T.I. Sergeeva, E.A. Baryshnikova, W. Zeiss, D. Möbius, O.V. Yescheoulova, S.P. Gromov, O.A. Fedorova, M.V. Alfimov, Mat. Sci. Eng. C 8-9 (1999) 469-473.

[9] S.Y. Zaitsev, E.A. Baryshnikova, T.I. Sergeeva, S.P. Gromov, O.A. Fedorova, O.V. Yescheoulova, M.A. Alfimov, S. Hacke, W. Zeiss, D. Möbius, Colloids Surf. 171 (2000) 283-290.

[10] S.Y. Zaitsev, E.A. Baryshnikova, T.I. Sergeeva, S.P. Gromov, O.A. Fedorova, O.V. Yescheoulova, M.V. Alfimov, S. Hacke, W. Zeiss, D. Möbius, Thin Solid Films 372 (2000) 230-236.

[11] T.I. Sergeeva, S.Y. Zaitsev, M.S. Tsarkova, S.P. Gromov, A.I. Vedernikov, M.V. Alfimov, T.S. Druzhinina, D. Möbius, J. Colloid Interface Sci. 265 (2003) 77-82.

[12] M. Orrit, D. Möbius, U. Lehmann, H. Meyer, J. Chen. Phys. 85 (1986) 4966-4979.

[13] T.I. Sergeeva, S.P. Gromov, A.I. Vedernikov, M.S. Kapichnikova, M.V. Alfimov, V.-T. Lieu, D. Möbius, M.S. Tsarkova, S.Y. Zaitsev, Colloids Surf. A, accepted for publication.

[14] M.T. Martín, I. Prieto, L. Camacho, D. Möbius, Langmuir 12 (1996) 6554-6560. 